\title{
Prognostic utility of T-wave alternans in a real-world population of patients with left ventricular dysfunction: the PREVENT-SCD study
}

\author{
Satoshi Shizuta - Kenji Ando - Masakiyo Nobuyoshi - Takanori Ikeda Hideaki Yoshino • \\ Shinichi Hiramatsu • Yukio Kazatani - Kohei Yamashiro • Katsunori Okajima - Teishi Kajiya • \\ Yoshinori Kobayashi · Takao Kato · Satoki Fujii • Kazuaki Mitsudo • Koichi Inoue $\cdot$ Hiroshi Ito \\ Yoshisumi Haruna · Takahiro Doi • Yukiko Nishio $\cdot$ Neiko Ozasa Kei Nishiyama • Toru Kita • \\ Takeshi Morimoto $\cdot$ Takeshi Kimura - For the PREVENT-SCD Investigators
}

Received: 9 May 2011/Accepted: 15 September 2011/Published online: 30 September 2011

(C) The Author(s) 2011. This article is published with open access at Springerlink.com

\begin{abstract}
Background The predictive value of T-wave alternans (TWA) for lethal ventricular tachyarrhythmia in patients with left ventricular (LV) dysfunction is controversial. Also, long-term arrhythmia risk of patients ineligible for the TWA test is unknown.

Methods This was a multicenter, prospective observational study of patients with LV ejection fraction $\leq 40 \%$ due to ischemic or non-ischemic cardiomyopathies, designed to evaluate the prognostic value of TWA for lethal ventricular tachyarrhythmia. The primary end point was a composite of sudden cardiac death, sustained rapid ventricular tachycardia (VT) or ventricular fibrillation
\end{abstract}

Investigators of the PRospective Evaluation of VENtricular Tachyarrhythmic events and Sudden Cardiac Death in patients with left ventricular dysfunction (PREVENT-SCD) study are listed in Appendix 1.

Electronic supplementary material The online version of this article (doi:10.1007/s00392-011-0368-2) contains supplementary material, which is available to authorized users.

S. Shizuta · Y. Haruna · T. Doi · Y. Nishio · N. Ozasa ·

K. Nishiyama $\cdot$ T. Kita $\cdot$ T. Kimura $(\bowtie)$

Department of Cardiovascular Medicine, Kyoto University

Graduate School of Medicine, 54 Kawahara-cho, Shougoin,

Sakyo-ku, Kyoto 606-8507, Japan

e-mail: taketaka@kuhp.kyoto-u.ac.jp

K. Ando · M. Nobuyoshi

Kokura Memorial Hospital, Kitakyushu, Japan

T. Ikeda $\cdot$ H. Yoshino

Kyorin University School of Medicine, Mitaka, Japan

S. Hiramatsu · Y. Kazatani

Ehime Prefectural Central Hospital, Matsuyama, Japan
(VF), and appropriate defibrillator therapy for rapid VT or VF.

Results Among 453 patients enrolled in the study, 280 (62\%) were eligible for the TWA test. TWA was negative in 82 patients (29\%), who accounted for $18 \%$ of the total population. The median of follow-up was 36 months. The 3 -year event-free rate for the primary end point was significantly higher in TWA-negative patients $(97.0 \%)$ than in TWA non-negative patients $(89.5 \%, P=0.037)$ and those ineligible for the TWA test $(84.4 \%, P=0.003)$. Multivariable analysis identified both non-negative TWA [hazard ratio (HR) 4.43 ; 95\% confidence interval (CI) $1.02-19.2 ; P=0.047)$ and ineligibility for the TWA test (HR 6.89; 95\% CI $1.59-29.9 ; P=0.010$ ) to be independent predictors of the primary end point.

Conclusions TWA showed high negative predictive ability for lethal ventricular tachyarrhythmia in patients with LV dysfunction, although the TWA-negative patients accounted for only $18 \%$ of the entire population. Those ineligible for the TWA test had the highest risk for lethal ventricular tachyarrhythmia.

K. Yamashiro $\cdot$ K. Okajima · T. Kajiya

Himeji Cardiovascular Center, Himeji, Japan

Y. Kobayashi · T. Kato

Nippon Medical School, Tokyo, Japan

S. Fujii · K. Mitsudo

Kurashiki Central Hospital, Kurashiki, Japan

K. Inoue $\cdot H$. Ito

Sakurabashi Watanabe Hospital, Osaka, Japan

T. Morimoto

Center for Medical Education, Kyoto University Graduate

School of Medicine, Kyoto, Japan 
Keywords Sudden death · Ventricular arrhythmia . Cardiomyopathy $\cdot \mathrm{T}$-wave alternans

\section{Introduction}

Recent randomized controlled trials have demonstrated the mortality benefit of an implantable cardioverter-defibrillator (ICD) over optimal medical therapy in patients with left ventricular (LV) dysfunction [1, 2]. The Multicenter Automatic Defibrillator Implantation Trial (MADIT) II showed $31 \%$ reduction in relative risk of mortality among patients with prior myocardial infarction and a LV ejection fraction (LVEF) $\leq 30 \%$ [1]. The Sudden Cardiac Death in Heart Failure Trial (SCD-HeFT) showed 23\% reduction in relative risk of mortality in patients with LVEF $\leq 35 \%$ and New York Heart Association (NYHA) class II-III congestive heart failure due to ischemic or nonischemic etiology [2].

Despite the significant reduction in mortality shown in MADIT II and SCD-HeFT, the absolute benefits were relatively small: $5.6 \%$ over 20 months and $7.2 \%$ over 5 years, respectively [1,2]. Because of concerns not only for the potential side effects of ICD [3-5] but also for the huge economic burden, further risk stratification to identify patients who are likely or unlikely to benefit from ICD seems to be necessary.

Microvolt-level T-wave alternans (TWA) in a bodysurface electrocardiogram (ECG) was proposed as a noninvasive method to evaluate risk of sudden cardiac death (SCD) or lethal ventricular tachyarrhythmia [6]. Although high negative predictive values of TWA have been reported [6-11], recent studies have reported conflicting data on the prognostic value of TWA [12-14]. Furthermore, TWA cannot be assessed in patients with persistent atrial fibrillation $(\mathrm{AF})$, ventricular pacing or exercise intolerance, who account for a sizable portion of the entire population of patients with LV dysfunction [15-17].

To address these issues, we have conducted a multicenter, prospective study assessing the predictive value of TWA for SCD or lethal ventricular tachyarrhythmia in a broad population of Japanese patients with LV dysfunction, including those ineligible for the TWA test.

\section{Methods}

Study design and patient enrollment

The Prospective Evaluation of Ventricular Tachyarrhythmic Events and Sudden Cardiac Death in Patients with Left Ventricular Dysfunction (PREVENT-SCD) study is a prospective multicenter registry of Japanese patients with
LV dysfunction, designed to evaluate the prognostic value of TWA and other potential arrhythmia risk factors for ventricular tachyarrhythmia. The study design is shown in Fig. 1.

Patients with LVEF $\leq 40 \%$ without treatable cardiac ischemia, who had NYHA class I-III heart failure, were eligible for the study. All patients had to be maintained on an optimal dose of angiotensin-converting enzyme inhibitors or angiotensin receptor blockers and beta-blockers before enrollment in the study unless these drugs were contraindicated or patients were intolerant.

Patients were excluded if they had a history of sustained ventricular fibrillation (VF) or ventricular tachycardia (VT), had previous ICD implantation, had NYHA class IV heart failure, had a myocardial infarction (MI) within the past month, had undergone coronary revascularization within the past 3 months, had treatable cardiac ischemia, had scheduled coronary revascularization or cardiac surgery, or had any condition other than cardiac disease with expectation of survival less than 1 year.

LV function was assessed by echocardiography using modified Simpson's method. When echocardiography was considered inappropriate due to poor images, then magnetic resonance imaging, angiography or radionuclide scanning was used to assess LV function.

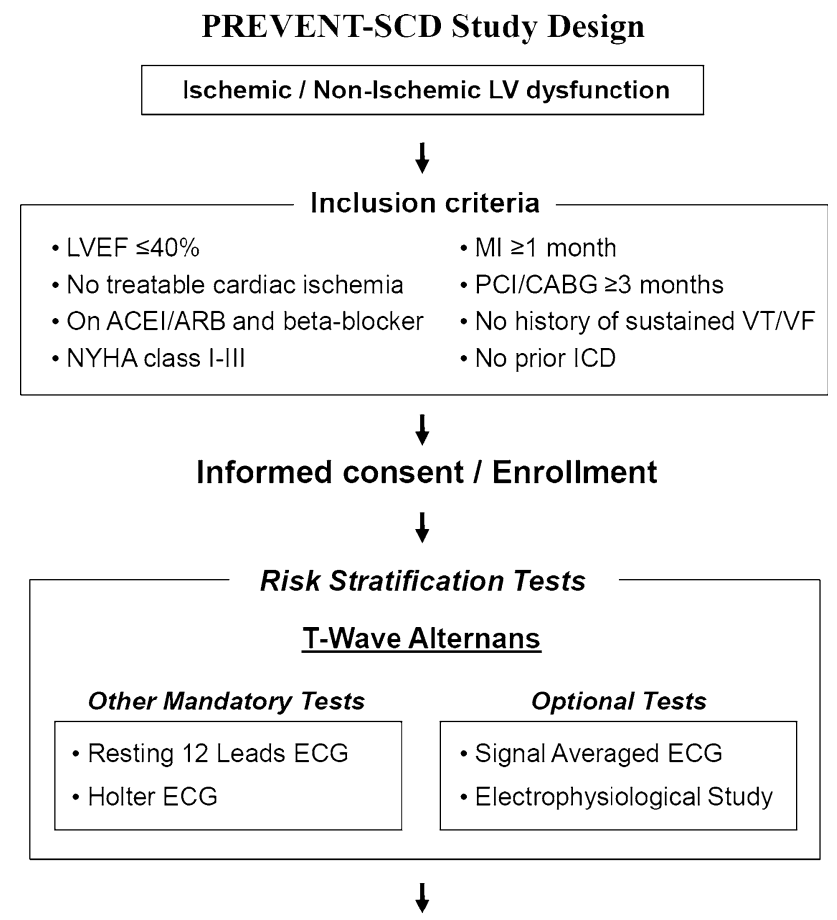

Therapeutic strategies left to discretion of each center

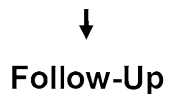

Fig. 1 Study design of the PREVENT-SCD study 
Ischemic cardiomyopathies were defined as LV dysfunction associated with a documented history of MI or severe narrowing of at least one of the three major coronary arteries. The others were classified as non-ischemic cardiomyopathies. Absence of treatable cardiac ischemia was confirmed by a stress test and/or coronary angiography.

Between June 2004 and February 2007, a total of 453 patients were enrolled from 38 centers in Japan (Appendix 1). Patients were scheduled to undergo TWA and other risk stratification tests, including 12-lead ECG and Holter ECG as mandatory tests and signal-averaged ECG and electrophysiological (EP) study as optional tests. Therapeutic strategies based on the results of the risk stratification tests were determined within 2 months of enrollment. The choice of therapeutic strategy, such as ICD implantation, was left to the discretion of the attending physicians. Device programming in ICD patients was not prespecified in this study. Case-specific programming was made at individual centers.

The relevant review boards or ethics committees in all participating centers approved the research protocol. Written informed consent was obtained from all patients.

\section{Risk stratification tests}

The TWA test was performed in all eligible patients. Patients underwent the TWA test while taking their regular medication, including beta-blockers. Careful skin preparation was performed and high-resolution electrodes were used to minimize noise. ECG leads were placed at the standard 12-lead positions and in an orthogonal X, Y and Z configuration. Measurements were made with a $\mathrm{CH} 2000$ or Heartwave system (Cambridge Heart Inc., Bedford, MA, USA). The result of the TWA test was automatically interpreted by the Alternans Report Classifier within the CH2000 or Heartwave system, reviewed by EP cardiologists at each individual center, and classified into positive, negative or indeterminate according to previously described criteria [18].

Because patients with indeterminate and positive TWA tests have been reported to have a similar arrhythmic or mortality risk [19], they were prospectively combined for analysis as 'non-negative' TWA tests. In this study, therefore, comparisons were made among patients with negative TWA, patients with non-negative (positive or indeterminate) TWA, and those ineligible for the TWA test.

Resting 12-lead ECG was evaluated for basic cardiac rhythm, heart rate, QRS duration, and QRS morphology. Holter ECG was evaluated for number of premature ventricular contractions (PVCs), as well as the presence or absence of non-sustained VT (NSVT). NSVT was defined as 3-30 PVCs at a rate $>100 / \mathrm{min}$.
Other potential arrhythmia risk predictors included age, sex, etiology of LV dysfunction, NYHA class, diabetes, hypertension, hyperlipidemia, AF, ventricular pacing, blood pressure, LVEF, LV end-diastolic dimension (LVEDD), mitral regurgitation, renal failure, anemia, brain natriuretic peptide (BNP) level and medication at the time of enrollment.

Follow-up and end points

All patients were followed in each participating center at least yearly after enrollment, either by hospital visits or telephone contact with the patients. When clinical events were suspected by telephone interview with the patients, they were confirmed by contact with the referring physicians. All ICD patients were followed at least every 6 months by hospital visits, and all ICD data were obtained by interrogation of the device. Clinical data were prospectively collected according to pre-specified definitions.

The prespecified primary end point of the study was a severe ventricular tachyarrhythmic event (SVTE), defined as a composite of SCD, sustained rapid-VT ( $\geq 188 / \mathrm{min})$ or VF, and appropriate ICD therapy for rapid-VT ( $\geq 188 / \mathrm{min})$ or VF. The secondary end points were all-cause death, cardiac death, and a ventricular tachyarrhythmic event (VTE) defined as a composite of SVTE, sustained VT $(<188 / \mathrm{min})$ and appropriate ICD therapy for VT $(<188 /$ $\min )$. SCD was defined as unexpected natural death occurring instantaneously or within $24 \mathrm{~h}$ of the onset of acute symptoms or signs, of which the cause was proved to be cardiac at necropsy or assumed to be cardiac because of clinical circumstances. Unwitnessed death of unknown cause was also regarded as SCD.

All of the primary and secondary events were adjudicated by a clinical events committee (Appendix 2).

\section{Statistical analyses}

All continuous variables are expressed as mean $\pm \mathrm{SD}$ or median. Statistical significance between the groups was assessed by Student's $t$ test for parametrically distributed continuous variables, the Wilcoxon rank sum test for nonparametrically distributed continuous variables, and Pearson's $\chi^{2}$ test for categorical variables.

Event-free survival was calculated by the Kaplan-Meier method. The significance of differences in event-free survival between groups was assessed with a log-rank test. Cox proportional hazards models were used to identify independent predictors of SVTE. Two dummy variables were created to reflect three levels of TWA status in the cohort: a negative test, a non-negative (positive or indeterminate) test, and no test (ineligible for the TWA test). The continuous variables were dichotomized by clinically 
meaningful reference values or median values. We first selected variables with $P$ values $<0.1$ in the univariable Cox models and then included them simultaneously in the multivariable models. Patients with missing values for any selected variable were excluded from the multivariable analysis. The independent predictors of SVTE were identified by the forward and backward stepwise methods with inclusion and exclusion $P$ values $<0.05$. Relative risks are expressed as hazard ratios (HRs) with $95 \%$ confidence intervals (CIs). All tests were 2 -sided, and a $P$ value $<0.05$ was considered statistically significant.

\section{Results}

Patient characteristics

The clinical characteristics of the 453 patients enrolled in the PREVENT-SCD study are listed in Table 1. The average age was $65.1 \pm 12.6$ years, and 367 (81\%) were male. The etiology was ischemic in 189 (42\%) and nonischemic in 264 (58\%). The mean LVEF was $29.2 \pm 7.4 \%$. Among the 189 ischemic patients, 170 (90\%) had had prior coronary revascularization.

TWA could be evaluated in 280 patients (62\%), and the results were negative in $82(29 \%)$, positive in $138(49 \%)$, and indeterminate in $60(21 \%)$. The TWA test was performed by an exercise test in 251 patients (90\%) and by atrial pacing in $29(10 \%)$. The reason for ineligibility for the TWA test was presence of AF in 65 patients $(38 \%)$, presence of ventricular pacing in $34(20 \%)$, presence of both $\mathrm{AF}$ and ventricular pacing in $12(7 \%)$, exercise intolerance in $50(29 \%)$, and other causes in $12(7 \%)$. The reason for the indeterminate TWA results was an inadequate heart rate increase in 29 patients $(48 \%)$, frequent ectopies in $13(22 \%)$, excessive noise in 10 (17\%), and other causes in 8 (13\%).

Resting 12-lead ECG was available in all patients. Forty-four percent of patients had QRS duration $\geq 120 \mathrm{~ms}$. Holter monitoring was performed in 433 patients $(95 \%)$. PVC $>10 / \mathrm{h}$ and NSVT were documented in 53 and $36 \%$, respectively.

ICD was implanted in 111 patients (25\%), 46 of which were in combination with cardiac resynchronization therapy.

Table 2 shows comparison of patients according to TWA status. TWA-non-negative patients had a higher prevalence of frequent PVCs than TWA-negative patients. Those ineligible for the TWA test were older, were associated with more severe heart failure symptoms, had higher BNP levels, had a wider QRS duration, and had a higher prevalence of AF or digitalis use as compared with TWA-negative patients. An ICD was used more often in TWA non-negative patients than in TWA-negative patients.

Long-term clinical outcome

During the median follow-up of 36 months (interquartile range 27-41 months), there were 47 first SVTEs, 70 first VTEs, and 68 deaths including 44 cardiac deaths (Table 3). Figure 2 shows the event-free curves for the primary end point of SVTE according to TWA status. The event-free rate for SVTE in TWA-negative patients was high, $100 \%$ at 1 year, $98.6 \%$ at 2 years, and $97.0 \%$ at 3 years. Both TWAnon-negative patients and those ineligible for the TWA test had significantly worse 3-year event-free rates for SVTE as compared with TWA-negative patients $(89.5 \%$ with $P=0.037$ and $84.4 \%$ with $P=0.003$, respectively). When TWA-non-negative patients were separated into TWAindeterminate and TWA-positive patients, the corresponding 3-year event-free rate for SVTE was $92.5 \%(P=0.22)$ and $88.1 \%(P=0.02)$, respectively. Among patients ineligible for the TWA test, those with AF, ventricular pacing or exercise intolerance had similar 3-year event-free rates for SVTE $(81.2,85.7$, and $86.5 \%$, respectively; $P=0.72)$.

Univariable and multivariable predictors of the primary end point are shown in Table 4. Multivariable analysis identified both non-negative TWA (HR 4.43; 95\% CI $1.02-19.2 ; P=0.047)$ and ineligibility for the TWA test (HR $6.89 ; 95 \%$ CI $1.59-29.9 ; P=0.010$ ) to be independent predictors of SVTE. When non-negative TWA was separated into indeterminate and positive TWA, the corresponding adjusted $\mathrm{HR}$ for indeterminate and positive TWA was 2.67 (95\% CI $0.48-14.7 ; P=0.26)$ and 5.36 (95\% CI 1.22-23.7; $P=0.027$ ), respectively. There was no significant interaction between TWA status and other clinical variables, including age, etiology of LV dysfunction, LVEF and QRS duration (Table 5).

Event-free curves for the secondary end points are shown in Fig. 3. Those ineligible for the TWA test had significantly worse event-free rates for all secondary end points as compared with TWA-negative patients with a HR of 3.15 for VTE (95\% CI 1.33-7.47), 3.57 for all-cause death (95\% CI 1.40-9.11), and 12.5 for cardiac death $(95 \%$ CI 1.69-91.9). TWA-non-negative patients had significantly worse cardiac survival than TWA-negative patients (HR 7.71; 95\% CI 1.04-57.7).

TWA negative patients had higher 3-year event-free rate for a composite of all-cause death and SVTE (91.9\%) as compared with TWA-non-negative patients $(81.7 \%$, $P=0.03)$ and those ineligible for the TWA test $(74.5 \%$, $P=0.0004)$. The 3 -year event-free rate for cardiac death or SVTE was 97.0, 86.2 $(P=0.008)$, and 78.9\% $(P<0.0001)$, respectively. 
Table 1 Patient characteristics

\begin{tabular}{|c|c|}
\hline \multicolumn{2}{|l|}{ Baseline characteristics } \\
\hline Age & $65.1 \pm 12.6$ \\
\hline Male & $367(81 \%)$ \\
\hline NYHA-III & $128(28 \%)$ \\
\hline Ischemic & $189(42 \%)$ \\
\hline Non-ischemic & $264(58 \%)$ \\
\hline \multicolumn{2}{|l|}{ Risk factors and co-morbidities } \\
\hline Prior MI & $180(40 \%)$ \\
\hline CABG/PCI & $170(38 \%)$ \\
\hline Stroke or TIA & $41(9 \%)$ \\
\hline Diabetes & $125(28 \%)$ \\
\hline Hypertension & $207(46 \%)$ \\
\hline Hyperlipidemia & $144(32 \%)$ \\
\hline $\mathrm{AF}$ & $120(26 \%)$ \\
\hline Persistent AF & $65(14 \%)$ \\
\hline Pacemaker & $56(12 \%)$ \\
\hline Systolic BP (mmHg) & $119 \pm 22$ \\
\hline Systolic BP $<100 \mathrm{mmHg}$ & $74(16 \%)$ \\
\hline Diastolic BP (mmHg) & $69 \pm 12$ \\
\hline Hemoglobin $<12$ g/dL & $108(24 \%)$ \\
\hline $\mathrm{eGFR}<40 \mathrm{~mL} / \mathrm{min} / 1.73 \mathrm{~m}^{2}$ & $90(20 \%)$ \\
\hline $\mathrm{BNP}(\mathrm{pg} / \mathrm{mL})$ & 211 \\
\hline $\mathrm{BNP} \geq 200 \mathrm{pg} / \mathrm{mL}$ & $228(52 \%)$ \\
\hline \multicolumn{2}{|l|}{ Echocardiography } \\
\hline $\operatorname{LVEF}(\%)^{\mathrm{a}}$ & $29.2 \pm 7.4$ \\
\hline $\mathrm{LVEF} \leq 30 \%$ & $228(50 \%)$ \\
\hline LVEDD (mm) & $62.3 \pm 8.9$ \\
\hline $\mathrm{LVEDD} \geq 70 \mathrm{~mm}$ & $90(20 \%)$ \\
\hline MR grade 3 or 4 & $58(13 \%)$ \\
\hline \multicolumn{2}{|l|}{ Medications } \\
\hline ACEIs or ARBs & $375(83 \%)$ \\
\hline Beta-blockers & $331(73 \%)$ \\
\hline Diuretics & $350(77 \%)$ \\
\hline Spironolactone & $236(52 \%)$ \\
\hline Digitalis & $139(31 \%)$ \\
\hline Amiodarone & $81(18 \%)$ \\
\hline Aspirin & $229(51 \%)$ \\
\hline Statin & $131(29 \%)$ \\
\hline \multicolumn{2}{|l|}{ Risk stratification tests } \\
\hline \multicolumn{2}{|l|}{ TWA $(N=280)$} \\
\hline Negative & $82(29 \%)$ \\
\hline Positive & $138(49 \%)$ \\
\hline Indeterminate & $60(21 \%)$ \\
\hline \multicolumn{2}{|c|}{ Resting 12-lead electrocardiogram $(N=453)$} \\
\hline Heart rate $(/ \min )$ & 71 \\
\hline QRS (ms) & 113 \\
\hline $\mathrm{QRS} \geq 120 \mathrm{~ms}$ & $199(44 \%)$ \\
\hline \multicolumn{2}{|l|}{ Holter monitoring $(N=433)$} \\
\hline $\mathrm{PVC}>10 / \mathrm{h}$ & $231(53 \%)$ \\
\hline NSVT & $157(36 \%)$ \\
\hline
\end{tabular}

Table 1 continued

Antiarrhythmic therapies

ICD

$111(25 \%)$

ICD with CRT

$46(10 \%)$

$A C E I s$ angiotensin-converting enzyme inhibitors, $A F$ atrial fibrillation, $A R B S$ angiotensin receptor blockers, $B N P$ brain natriuretic peptide, $B P$ blood pressure, $C A B G$ coronary artery bypass grafting, $C R T$ cardiac resynchronization therapy, $e G F R$ estimated glomerular filtration rate, $I C D$ implantable cardioverter-defibrillator, $L V E D D$ left ventricular end-diastolic dimension, $L V E F$ left ventricular ejection fraction, $M I$ myocardial infarction, $M R$ mitral regurgitation, NSVT non-sustained ventricular tachycardia, NYHA New York Heart Association, $P C I$ percutaneous coronary intervention, $P V C$ premature ventricular contraction, TIA transient ischemic attack, TWA T-wave alternans

${ }^{\text {a }}$ Measured by modified Simpson's method. For 33 patients with poor echo images, magnetic resonance imaging was used in 15 patients, angiography in 15 patients, and radionuclide scanning in 3 patients

\section{Discussion}

The present study focused on the predictive value of TWA for lethal ventricular tachyarrhythmia in a broad population of patients with LV dysfunction. The event-free rate for SVTE in TWA-negative patients was excellent, $100 \%$ at 1 year, $98.6 \%$ at 2 years, and $97.0 \%$ at 3 years, which may suggest that TWA-negative patients can be safely excluded as ICD candidates. When those ineligible for the TWA test were taken into account, however, the TWA-negative patients accounted for only $18 \%$ of the entire study population. Thus, despite the strikingly high negative predictive value of TWA for SVTE, the safety assurance afforded by a negative result of the TWA test was applicable to only a limited proportion of patients with LV dysfunction.

The other important finding of this study was the poor long-term prognosis of patients ineligible for the TWA test. Ineligibility for the TWA test was associated with highest risk not only for the primary end point of SVTE but for all of the secondary end points, including VTE, all-cause death and cardiac death.

All previous TWA studies have excluded patients ineligible for the TWA test. Exclusion of those patients might be associated with considerable selection bias toward patients with good exercise capacity and those without AF or ventricular pacing. Although the prevalence of negative TWA among eligible patients with LV dysfunction has been reported to be $22-37 \%$ [8-14], a value coincident with the $29 \%$ observed in the present study, the proportion of TWA-negative patients among all patients with LV dysfunction has not previously been established. In addition, the long-term arrhythmia and mortality risk of patients ineligible for the TWA test has been uncertain. To the best of our knowledge, our study is the first multicenter study evaluating the prognostic utility of TWA in a real- 
Table 2 Comparison of patients according to TWA status

\begin{tabular}{llll}
\hline & TWA-negative $(N=82)$ & TWA-non-negative $(N=198)$ & Ineligible for TWA $(N=173)$ \\
\hline Age & $60.5 \pm 15.4$ & $63.8 \pm 12.3$ & $68.7 \pm 10.2^{\dagger}$ \\
Ischemic & $41(50 \%)$ & $94(57 \%)$ & $54(31 \%)^{\dagger}$ \\
NYHA-III & $13(16 \%)$ & $34(17 \%)$ & $81(47 \%)^{\dagger}$ \\
AF & $6(7 \%)$ & $21(11 \%)$ & $93(54 \%)^{\dagger}$ \\
Systolic BP $(\mathrm{mmHg})$ & $120 \pm 23$ & $120 \pm 20$ & $117 \pm 23$ \\
Hemoglobin $(\mathrm{g} / \mathrm{dL})$ & $13.6 \pm 1.8$ & $13.4 \pm 3.2$ & $13.3 \pm 2.2$ \\
BNP $(\mathrm{pg} / \mathrm{mL})$ & 155 & 174 & $286^{\dagger}$ \\
LVEF $(\%)$ & $29.8 \pm 7.2$ & $29.2 \pm 7.2$ & $28.8 \pm 7.6$ \\
LVEDD $(\mathrm{mm})$ & $61.2 \pm 8.0$ & $63.2 \pm 8.9$ & $61.7 \pm 9.2$ \\
MR grade 3 or 4 & $8(10 \%)$ & $23(12 \%)$ & $27(16 \%)$ \\
Digitalis & $21(26 \%)$ & $41(21 \%)$ & $77(45 \%)$ \\
QRS $(\mathrm{ms})$ & 103 & 109 & $127^{\dagger}$ \\
PVC $>10 / \mathrm{h}$ & $31(39 \%)$ & $105(54 \%)^{*}$ & $95(60 \%)^{\dagger}$ \\
NSVT & $25(31 \%)$ & $66(34 \%)$ & $66(42 \%)$ \\
ICD & $12(15 \%)$ & $60(30 \%)^{*}$ & $39(23 \%)$ \\
\hline
\end{tabular}

Abbreviations as in Table 1

* $P<0.05 ;{ }^{\dagger} P<0.005$ in comparison with TWA-negative group

Table 3 Primary and secondary end points during follow-up

\begin{tabular}{ll}
\hline First SVTE & $47(10.4 \%)$ \\
SCD & $15^{\mathrm{a}}$ \\
Sustained rapid-VT/VF $(\geq 188 / \mathrm{min})$ & 8 \\
ICD therapy for rapid-VT/VF $(\geq 188 / \mathrm{min})$ & 24 \\
First VTE & $70(15.5 \%)$ \\
SVTE & $31^{\mathrm{b}}$ \\
Sustained VT $(<188 / \mathrm{min})$ & 3 \\
ICD therapy for VT $(<188 / \mathrm{min})$ & 36 \\
Death & $68(15.0 \%)$ \\
Cardiac death & $44(9.7 \%)$ \\
SCD & $17(3.8 \%)$ \\
\hline
\end{tabular}

$\overline{S C D}$ sudden cardiac death, SVTE severe ventricular tachyarrhythmic event, $V F$ ventricular fibrillation, $V T$ ventricular tachycardia, VTE ventricular tachyarrhythmic event

${ }^{\text {a }}$ Out of 17 SCDs, 15 were first SVTE

b Out of 47 SVTEs, 31 were first VTE

world population of LV dysfunction patients, including those ineligible for the TWA test. TWA showed high negative predictive ability for lethal ventricular tachyarrhythmia and cardiac mortality, but the proportion of TWA-negative patients was less than one-fifth of the entire study population. Those ineligible for the TWA test had highest arrhythmia and mortality risk.

To date, seven multicenter studies assessing the predictive value of TWA for lethal ventricular tachyarrhythmia in patients with LV dysfunction have been reported (Supplementary Table) [8-14]. Four of those studies reported the significant prognostic utility of TWA with

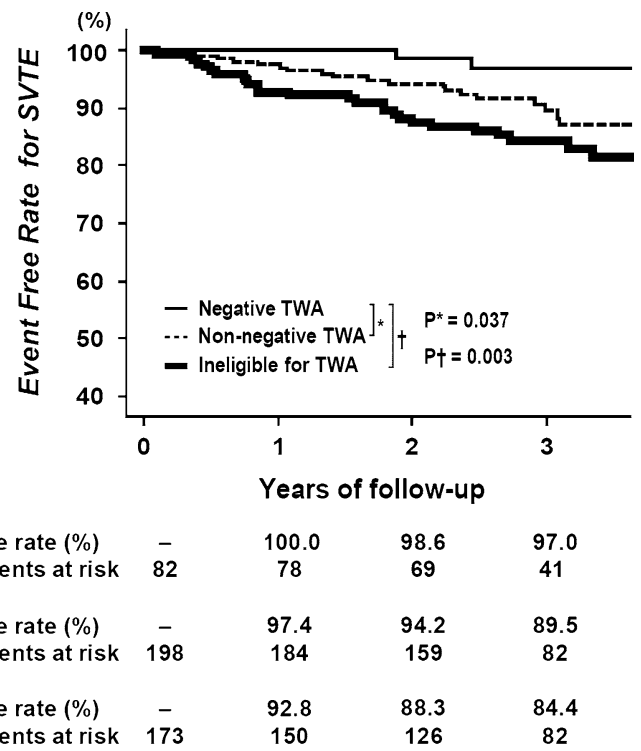

Fig. 2 Event-free curves for the primary end point of SVTE according to TWA status

high negative predictive values for arrhythmic events [811], as shown in our study. However, the other three more recent studies have reported conflicting data on the predictive value of TWA [12-14]. The Microvolt T Wave Alternans Testing for Risk Stratification of Post-Myocardial Infarction Patients (MASTER) trial and the SCDHeFT substudy showed no difference in arrhythmic event rates between TWA-negative and TWA-non-negative patients $[12,13]$. Furthermore, in the Alternans Before Cardioverter Defibrillator (ABCD) trial, TWA showed 
Table 4 Univariable and multivariable predictors of SVTE

$C I$ confidence interval, $H R$ hazard ratio. Other abbreviations as in Table 1

\begin{tabular}{|c|c|c|c|c|}
\hline \multirow[t]{2}{*}{ Variables } & \multicolumn{2}{|l|}{ Univariable analysis } & \multicolumn{2}{|l|}{ Multivariable analysis } \\
\hline & Unadjusted HR (95\% CI) & $P$ value & Adjusted HR (95\% CI) & $P$ value \\
\hline \multicolumn{5}{|l|}{ TWA } \\
\hline Ineligible & $6.69(1.59-28.2)$ & 0.010 & $6.89(1.59-29.9)$ & 0.010 \\
\hline Non-negative & $4.15(0.97-17.8)$ & 0.056 & $4.43(1.02-19.2)$ & 0.047 \\
\hline MR grade 3 or 4 & $4.69(2.56-8.61)$ & $<0.001$ & $3.61(1.80-7.27)$ & $<0.001$ \\
\hline Age $<65$ years & $1.70(0.95-3.02)$ & 0.072 & $2.48(1.32-4.68)$ & 0.005 \\
\hline Systolic BP $<100 \mathrm{mmHg}$ & $2.91(1.58-5.38)$ & 0.001 & $2.43(1.25-4.73)$ & 0.009 \\
\hline $\mathrm{PVC}>10 / \mathrm{h}$ & $3.01(1.52-5.95)$ & 0.002 & $2.35(1.16-4.76)$ & 0.017 \\
\hline NSVT & $2.56(1.41-4.64)$ & 0.002 & - & - \\
\hline LVEDD $\geq 70 \mathrm{~mm}$ & $2.22(1.21-4.06)$ & 0.010 & - & - \\
\hline $\mathrm{BNP} \geq 200 \mathrm{pg} / \mathrm{mL}$ & $2.13(1.14-3.96)$ & 0.017 & - & - \\
\hline $\mathrm{QRS} \geq 120 \mathrm{~ms}$ & $1.91(1.07-3.40)$ & 0.029 & - & - \\
\hline $\mathrm{AF}$ & $1.87(1.04-3.37)$ & 0.037 & - & - \\
\hline Digitalis & $1.78(1.00-3.16)$ & 0.050 & - & - \\
\hline $\mathrm{LVEF} \leq 35 \%$ & $2.12(0.90-4.96)$ & 0.060 & - & - \\
\hline Hemoglobin $<12$ g/dL & $1.75(0.95-3.23)$ & 0.087 & - & - \\
\hline
\end{tabular}

limited predictive ability for lethal ventricular tachyarrhythmia. The difference in the primary event rates between TWA-negative and TWA-non-negative patients was significant up to 9 months, but no longer after 12 months [14].

There are several possible explanations for these conflicting results. First, among the studies there were substantial differences in TWA protocol, which might have influenced the predictive accuracy of the TWA test. In four studies including MASTER, SCD-HeFT and ABCD, betablockers were withheld for $\geq 24 \mathrm{~h}$ before the TWA test in order to reduce indeterminate TWA results due to an inadequate heart rate increase during exercise. However, Klingenheben et al. [20] reported that metoprolol and sotalol decreased TWA amplitude by 35 and $38 \%$, respectively. Also, in a study by Rashba et al. [21], intravenous beta-blocker of esmolol reduced the number of positive TWA tests by $50 \%$. These data suggest that betablockers should be continued at the time of the TWA test. In a recent meta-analysis of TWA studies by Chan et al. [22], non-negative TWA was associated with a fivefold increased risk for ventricular tachyarrhythmia as compared with negative TWA in studies where beta-blockers were continued during the TWA test, while only a much weaker association was observed in studies where beta-blockers were withheld prior to the TWA test.

Second, it may be that previous studies did not exclude patients with treatable cardiac ischemia, which could affect the prognostic value of the TWA test. Patients with heavy ischemic burden are considered to have higher risk for a future coronary event, which is often associated with lethal ventricular tachyarrhythmia in patients with $\mathrm{LV}$ dysfunction
[23]. In the present study, coronary artery disease was well treated among ischemic patients, none of whom had treatable cardiac ischemia.

Third, the prevalence of ICD use was much higher in MASTER and ABCD, 100 and 87\%, respectively, as compared with $25 \%$ in the present study. The use of appropriate ICD therapy for ventricular tachyarrhythmia as a surrogate end point for SCD may overestimate arrhythmic events, by counting potential self-terminating tachycardia as events [24]. Thus, a higher prevalence of ICD use may lead to a higher possibility of overestimating arrhythmic events.

The low prevalence of ICD use in the present study was largely due to the fact that reimbursement officially covers ICDs only for secondary prevention of SCD in Japan. Because patient enrollment of this study started in June 2004, when the results of MADIT II and SCD-HeFT had been already published or announced, therapeutic strategies (i.e., whether to implant ICD or not) were left to the discretion of the attending physician. As a result, ICD patients were much sicker than Non-ICD patients in terms of lower systolic blood pressure, higher BNP level, lower LVEF, larger LVEDD, wider QRS duration, lower prevalence of negative-TWA, and higher prevalence of moderate to severe mitral regurgitation, PVC $>10 / \mathrm{h}$, or NSVT (data not shown).

The TWA test was initially developed as risk stratification test for lethal ventricular tachyarrhythmia mainly in ischemic patients [6-8], but recent studies indicate that predictive values of TWA might be higher in non-ischemic patients than in ischemic patients [9, 13]. Consistently, in the present study, the negative predictive value of TWA for SVTE was $100 \%$ in non-ischemic patients, as compared 
Table 5 Risk of SVTE according to TWA status in patient subgroups
* $P$ for interaction between TWA and each variable based on difference in probabilities of events; TWA-negative versus TWA-non-negative (left) and TWA-negative versus ineligible for TWA (right)

a Indeterminate because of no events in TWA-negative group

\begin{tabular}{|c|c|c|c|}
\hline Subgroups & Events/n $(\%)$ & Hazard ratio $(95 \% \mathrm{CI})$ & Interaction $P^{*}$ \\
\hline Age & & & $0.09 / 0.20$ \\
\hline \multicolumn{4}{|l|}{$<65$ years } \\
\hline TWA-negative & $1 / 46(2.2 \%)$ & & \\
\hline TWA-non-negative & $13 / 91(14.3 \%)$ & $7.69(1.01-58.8)$ & \\
\hline Ineligible for TWA & $12 / 58(20.7 \%)$ & $11.21(1.46-86.2)$ & \\
\hline \multicolumn{4}{|l|}{$\geq 65$ years } \\
\hline TWA-negative & $1 / 36(2.8 \%)$ & & \\
\hline TWA-non-negative & $6 / 107(5.6 \%)$ & $1.92(0.23-16.0)$ & \\
\hline Ineligible for TWA & $14 / 115(12.2 \%)$ & $4.44(0.58-33.8)$ & \\
\hline Etiology of LV dysfunction & & & $0.19 / 0.10$ \\
\hline \multicolumn{4}{|l|}{ Ischemic } \\
\hline TWA-negative & $2 / 41(4.9 \%)$ & & \\
\hline TWA-non-negative & $8 / 94(8.5 \%)$ & $1.87(0.40-8.79)$ & \\
\hline Ineligible for TWA & $6 / 54(11.1 \%)$ & $2.38(0.48-11.8)$ & \\
\hline \multicolumn{4}{|l|}{ Non-ischemic } \\
\hline TWA-negative & $0 / 41(0 \%)$ & & \\
\hline TWA-non-negative & $11 / 104(10.6 \%)$ & $-{ }^{\mathrm{a}}$ & \\
\hline Ineligible for TWA & $20 / 119(16.8 \%)$ & $-^{\mathrm{a}}$ & \\
\hline LVEF & & & $0.19 / 0.05$ \\
\hline \multicolumn{4}{|l|}{$\leq 35 \%$} \\
\hline TWA-negative & $1 / 63(1.6 \%)$ & & \\
\hline TWA-non-negative & $17 / 160(10.6 \%)$ & $7.06(0.94-53.09)$ & \\
\hline Ineligible for TWA & $23 / 130(17.7 \%)$ & $12.52(1.69-92.74)$ & \\
\hline \multicolumn{4}{|l|}{$>35 \%$} \\
\hline TWA-negative & $1 / 19(5.3 \%)$ & & \\
\hline TWA-non-negative & $2 / 38(5.3 \%)$ & $1.06(0.14-12.51)$ & \\
\hline Ineligible for TWA & $3 / 43(7.0 \%)$ & $1.30(0.10-11.71)$ & \\
\hline QRS duration & & & $0.46 / 0.05$ \\
\hline \multicolumn{4}{|l|}{$\geq 120 \mathrm{~ms}$} \\
\hline TWA-negative & $0 / 24(0 \%)$ & & \\
\hline TWA-non-negative & $7 / 72(9.7 \%)$ & $-^{\mathrm{a}}$ & \\
\hline Ineligible for TWA & $20 / 103(19.6 \%)$ & $-{ }^{\mathrm{a}}$ & \\
\hline \multicolumn{4}{|l|}{$<120 \mathrm{~ms}$} \\
\hline TWA-negative & $2 / 58(3.4 \%)$ & & \\
\hline TWA-non-negative & $12 / 126(9.5 \%)$ & $2.91(0.51-12.6)$ & \\
\hline Ineligible for TWA & $6 / 70(8.6 \%)$ & $2.51(0.65-13.0)$ & \\
\hline
\end{tabular}

with $95.1 \%$ in ischemic patients, although the difference was not statistically significant (Table 5).

Because follow-up duration of previous studies reporting high predictive values of TWA ranged from 17 to 20 months [6-11], it has been uncertain whether the prognostic utility of TWA remains robust beyond 2 years. In the present study, the median of follow-up was 36 months, which was the longest among the multicenter TWA studies. The 3-year event-free rates for SVTE and cardiac death in TWA-negative patients were extremely high, 97.0 and $98.6 \%$, respectively. Our data suggest that the predictive value of TWA remains robust over 3 years.

\section{Limitations}

The present study has several important limitations. First, the number of patients enrolled in this study was not large enough, considering the broad spectrum of its patient population. Second, the number of patients screened and excluded in this study was not recorded. Considering the relatively slow rate of patient enrolment, there may be some selection bias in our study population. Third, inclusion of appropriate ICD therapy in the composite primary end point may overestimate arrhythmic events, as discussed above. However, the prevalence of ICD use in the 
Fig. 3 Event-free curves for the secondary end points according to TWA status. Event-free curves for VTE (a), all-cause death (b) and cardiac death (c)
(A)
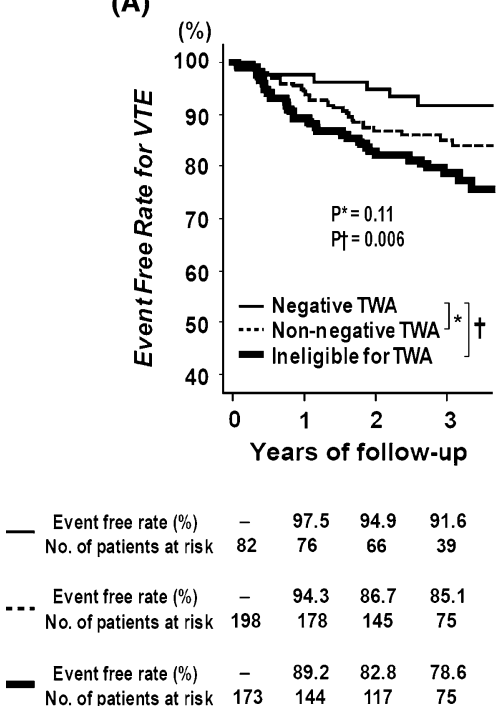

(B)
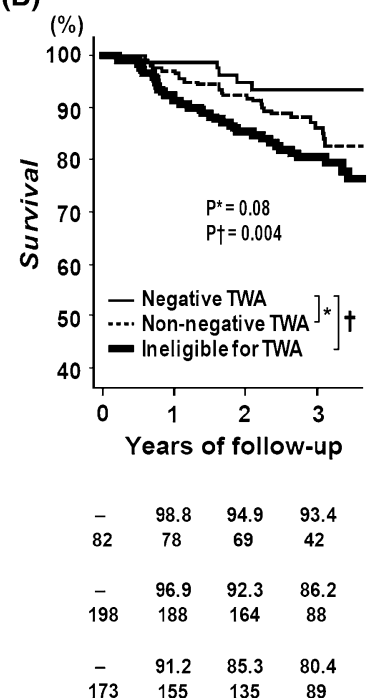

(C)

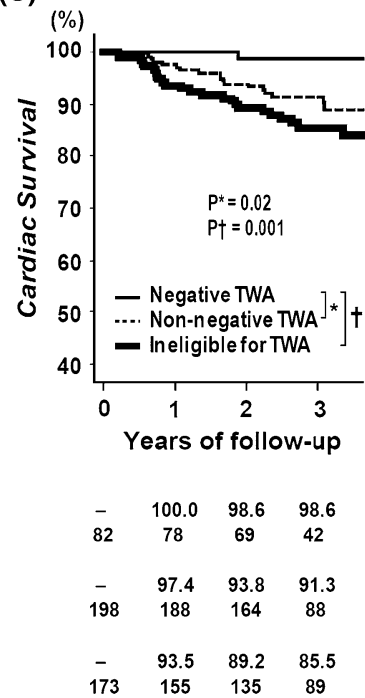

present study was relatively low as compared with other recent studies. Fourth, the rate of self-terminating ventricular tachycardia in patients without an ICD could not be determined. Fifth, device programming in ICD patients was not prespecified in this study. All intra-cardiac ECGs of ICD therapies were, however, reviewed by the clinical events committee. Finally, the results of the TWA test were determined at each participating center without a core laboratory so that the data would more closely represent general clinical practice. Conversely, there may be some heterogeneity in the interpretation of the TWA test results.

\section{Conclusions}

The present study demonstrated high negative predictive ability of TWA for lethal ventricular tachyarrhythmia in a real-world Japanese population of LV dysfunction patients during the median follow-up of 3 years. However, the TWA-negative patients accounted for only $18 \%$ of the entire population, including those ineligible for the TWA test. Thus, despite the strikingly high negative predictive value of TWA for lethal ventricular arrhythmia, the safety assurance given by a negative result of the TWA test was applicable to only a limited proportion of patients with $\mathrm{LV}$ dysfunction.

Those ineligible for the TWA test were associated with highest risk for lethal ventricular tachyarrhythmia, as well as highest overall and cardiac mortality.

Our study warrants further investigation with a larger patient population and a longer follow-up period.

Acknowledgments We are indebted to the clinical research coordinators (Appendix 3) for their invaluable contributions to the data collection. This study was supported by Medtronic Japan Co., Ltd.;
Fukuda Denshi; Roche Diagnostics K. K.; Dainippon Sumitomo Pharma Co., Ltd.; and Mitsubishi Kagaku Iatron, Inc. The study sponsors were not involved in the study design; in the collection, analysis, and interpretation of data; in the writing of the report; or in the decision to submit the article for publication.

Conflict of interest S.S. has received research support and honoraria from Medtronic Japan Co., Ltd. and honoraria from Fukuda Denshi. K.Y. has received honoraria and consultation fee from Medtronic Japan Co., Ltd. T. Ka has received consultation fee from Dainippon Sumitomo Pharma Co., Ltd. T. Ki has received research support from Medtronic Japan Co., Ltd. and Fukuda Denshi. Others: none declared.

Open Access This article is distributed under the terms of the Creative Commons Attribution Noncommercial License which permits any noncommercial use, distribution, and reproduction in any medium, provided the original author(s) and source are credited.

\section{Appendix 1: List of participating centers and investigators}

The following centers and investigators participated in the PREVENT-SCD study (listed in alphabetical order according to center):

Ehime Prefectural Central Hospital: Yukio Kazatani, Shinichi Hiramatsu, Tsuyoshi Matsunaka, Sumiko Sato; Fujita Health University Hospital: Hitoshi Hishida, Eiichi Watanabe; Funabashi Municipal Medical Center: Shun Ozawa, Masayuki Inagaki; Himeji Cardiovascular Center: Teishi Kajiya, Kohei Yamashiro, Katsunori Okajima; Hirosaki University School of Medicine \& Hospital: Ken Okumura, Atsushi Iwasa, Masaomi Kimura, Hyogo Prefectural Amagasaki Hospital: Yoshiki Takatsu, Ryoji Taniguchi; International Medical Center of Japan: Mitsuo Kashida, Osamu Okazaki; Kagoshima University Medical 
And Dental Hospital: Chuwa Tei, Shuichi Hamasaki, Kobe University Hospital: Mitsuhiro Yokoyama, Akihiro Yoshida; Kokura Memorial Hospital: Masakiyo Nobuyoshi, Kenji Ando, Hitoshi Okabayashi, Jyota Nakano; Kurashiki Central Hospital: Kazuaki Mitsudo Satoki Fujii; Kurume University Hospital: Teruhisa Yoshida, Yasutsugu Nagamoto, Eiichi Takii; Kyorin University Hospial: Hideaki Yoshino, Takanori Ikeda; Kyoto University Graduate School of Medicine: Takeshi Kimura, Satoshi Shizuta, Yoshisumi Haruna, Takahiro Doi, Yukiko Nishio, Neiko Ozasa, Kei Nishiyama, Toru Kita, Takeshi Morimoto; Kyoto-Katsura Hospital: Shigeru Nakamura, Yoshihisa Enjoji; Mitsubishi Kyoto Hospital: Shinji Miki, Yutaka Kono; Nagoya University Hospital: Toyoaki Murohara, Yasuya Inden; National Defense Medical College Hospital: Fumitaka Ohsuzu, Koh Arakawa; Niigata University Medical \& Dental Hospital: Yoshifusa Aizawa, Hiroshi Furushima; Nippon Medical School Hospital: Takao Kato, Yoshinori Kobayashi; Oita University Hospital: Tetsunori Saikawa, Naohiko Takahashi; Okayama University Hospital: Tohru Ohe, Satoshi Nagase; Ome Municipal General Hospital: Kenichiro Otomo; Sakurabashi Watanabe Hospital: Hiroshi Ito, Koichi Inoue; Shiga University of Medical Science Hospital: Minoru Horie, Takashi Yamamoto, Masanori Fujii, Makoto Ito; Showa University Fujigaoka Hospital: Youichi Takeyama, Yukei Higashi; St. Marianna University School of Medicine Hospital: Fumihiko Miyake, Ryouji Kishi, Kiyoshi Nakazawa; St. Marianna University School of Medicine, Yokohama City Seibu Hospital: Haruki Musha, Osamu Miyatsu; Teikyo University Hospital: Takaaki Isshiki, Taiji Furukawa; Teikyo University School of Medicine University Hospital, Mizonokuchi: Tomoyuki Kunishima; The Hospital of Hyogo College of Medicine: Mitsumasa Ohyanagi, Hiroki Shimizu; Toho University Ohashi Medical Center: Kaoru Sugi, Takao Sakata; Tokyo Medical and Dental University Hospital Faculty of Medicine: Mitsuaki Isobe, Kenzo Hirao; Tokyo Metropolitan Hiroo Hospital: Harumizu Sakurada, Tsuyoshi Sakai, Hidetaka Okazaki, Akiko Tatsumoto; Toyama University Hospital: Hiroshi Inoue, Tomoki Kameyama; Tsuchiya General Hospital: Yasuhiko Hayashi, Yuji Muraoka; Yamaguchi University Hospital: Masunori Matsuzaki, Akihiko Shimizu; Yokohama City University Medical Center: Kazuo Kimura, Toshiaki Ebina.

\section{Appendix 2: Members of the clinical events committee}

Kenji Ando, Takanori Ikeda, Yoshinori Kobayashi, Satoshi Shizuta.

\section{Appendix 3: List of clinical research coordinators}

Asuka Saeki, Hiromi Yoshida, Yuko Yamamoto, Kumiko Kitagawa, Misato Yamauchi, Naoko Okamoto, Yumika Fujino, Izumi Miki, Yuki Sato, Yoko Fujiki, Rei Fujita, Miya Hanazawa, Miho Hayashikawa, Chikako Hibi, Takami Hiraoka, Sachiko Maeda, Saeko Minematsu, Ayu Motofusa, Satoko Nishida, Hitomi Sasae, Yayoi Sugimoto, Emi Takinami, Saori Tezuka, Ayumi Yamamoto, Mai Yoshimoto.

\section{References}

1. Moss AJ, Zareba W, Hall WJ, Klein H, Wilber DJ, Cannom DS et al (2002) Prophylactic implantation of a defibrillator in patients with myocardial infarction and reduced ejection fraction. N Engl J Med 346:877-883

2. Bardy GH, Lee KL, Mark DB, Poole JE, Packer DL, Boineau R et al (2005) Amiodarone or an implantable cardioverter-defibrillator for congestive heart failure. N Engl J Med 352:225-237

3. van Rees JB, Borleffs CJ, de Bie MK, Stijnen T, van Erven L, Bax JJ et al (2011) Inappropriate implantable cardioverter-defibrillator shocks: incidence, predictors, and impact on mortality. J Am Coll Cardiol 57:556-562

4. Lenz C, Dietze T, Möller M, Schöbel W, Wicke J, Kellner HJ, Gradaus R, Neuzner J (2009) Incessant ventricular tachycardia, refractory to catheter ablation, in an ICD patient terminated by ICD lead extraction: a case report. Clin Res Cardiol. 98:803-805

5. Herold J, Guenther M, Strasser RH, Braun M (2009) Twiddler's syndrome in an adolescent patient with ICD during neurological and physical rehabilitation. Clin Res Cardiol. 98:137-139

6. Rosenbaum DS, Jackson LE, Smith JM, Garan H, Ruskin JN, Cohen RJ (1994) Electrical alternans and vulnerability to ventricular arrhythmias. N Engl J Med 330:235-241

7. Klingenheben T, Zabel M, D'Agostino RB, Cohen RJ, Hohnloser SH (2000) Predictive value of T-wave alternans for arrhythmic events in patients with congestive heart failure. Lancet 356:651-652

8. Hohnloser SH, Ikeda T, Bloomfield DM, Dabbous OH, Cohen RJ (2003) T-wave alternans negative coronary patients with low ejection and benefit from defibrillator implantation. Lancet 362:125-126

9. Bloomfield DM, Bigger JT, Steinman RC, Namerow PB, Parides MK, Curtis AB et al (2006) Microvolt T-wave alternans and the risk of death or sustained ventricular arrhythmias in patients with left ventricular dysfunction. J Am Coll Cardiol 47:456-463

10. Chow T, Kereiakes DJ, Bartone C, Booth T, Schloss EJ, Waller T et al (2006) Prognostic utility of microvolt T-wave alternans in risk stratification of patients with ischemic cardiomyopathy. J Am Coll Cardiol 47:1820-1827

11. Salerno-Uriarte JA, De Ferrari GM, Klersy C, Pedretti RF, Tritto M, Sallusti L et al (2007) Prognostic value of T-wave alternans in patients with heart failure due to nonischemic cardiomyopathy: results of the ALPHA Study. J Am Coll Cardiol 50:1896-1904

12. Chow T, Kereiakes DJ, Onufer J, Woelfel A, Gursoy S, Peterson BJ et al (2008) Does microvolt T-wave alternans testing predict ventricular tachyarrhythmias in patients with ischemic cardiomyopathy and prophylactic defibrillators? The MASTER (Microvolt T Wave Alternans Testing for Risk Stratification of 
Post-Myocardial Infarction Patients) trial. J Am Coll Cardiol 52:1607-1615

13. Gold MR, Ip JH, Costantini O, Poole JE, McNulty S, Mark DB et al (2008) Role of microvolt T-wave alternans in assessment of arrhythmia vulnerability among patients with heart failure and systolic dysfunction: primary results from the T-wave alternans sudden cardiac death in heart failure trial substudy. Circulation. 118:2022-2028

14. Costantini O, Hohnloser SH, Kirk MM, Lerman BB, Baker JH II, Sethuraman B et al (2009) The ABCD (Alternans Before Cardioverter Defibrillator) Trial: strategies using T-wave alternans to improve efficiency of sudden cardiac death prevention. J Am Coll Cardiol 53:471-479

15. Maisel WH, Stevenson LW (2003) Atrial fibrillation in heart failure: epidemiology, pathophysiology, and rationale for therapy. Am J Cardiol 91:2D-8D

16. Sandhu R, Bahler RC (2004) Prevalence of QRS prolongation in a community hospital cohort of patients with heart failure and its relation to left ventricular systolic dysfunction. Am J Cardiol 93:244-246

17. Kokkinos PF, Choucair W, Graves P, Papademetriou V, Ellahham S (2000) Chronic heart failure and exercise. Am Heart J 140:21-28

18. Bloomfield DM, Hohnloser SH, Cohen RJ (2002) Interpretation and classification of microvolt $\mathrm{T}$ wave alternans tests. J Cardiovasc Electrophysiol 13:502-512
19. Kaufman ES, Bloomfield DM, Steinman RC, Namerow PB, Costantini O, Cohen RJ et al (2006) "Indeterminate" microvolt T-wave alternans tests predict high risk of death or sustained ventricular arrhythmias in patients with left ventricular dysfunction. J Am Coll Cardiol 48:1399-1404

20. Klingenheben T, Grönefeld G, Li YG, Hohnloser SH (2001) Effect of metoprolol and d,1-sotalol on microvolt-level T-wave alternans. Results of a prospective, double-blind, randomized study. J Am Coll Cardiol 38:2013-2019

21. Rashba EJ, Cooklin M, MacMurdy K, Kavesh N, Kirk M, Sarang $S$ et al (2002) Effects of selective autonomic blockade on T-wave alternans in humans. Circulation. 105:837-842

22. Chan PS, Gold MR, Nallamothu BK (2010) Do beta-blockers impact microvolt $\mathrm{T}$-wave alternans testing in patients at risk for ventricular arrhythmias? A meta-analysis. J Cardiovasc Electrophysiol 21(9):1009-1014

23. Hohnloser SH, Kuck KH, Dorian P, Roberts RS, Hampton JR, Hatala $R$ et al (2004) Prophylactic use of an implantable cardioverter-defibrillator after acute myocardial infarction. N Engl J Med 351:2481-2488

24. Hohnloser SH, Ikeda T, Cohen RJ (2009) Evidence regarding clinical use of microvolt T-wave alternans. Heart Rhythm. 6:S36S44 